University of Nebraska - Lincoln

DigitalCommons@University of Nebraska - Lincoln

1970

\title{
Experimental Evidence for the Mechanism of Charge Transfer into Continuum States
}

G. B. Crooks

University of Nebraska - Lincoln

M. Eugene Rudd

University of Nebraska - Lincoln, erudd@unl.edu

Follow this and additional works at: https://digitalcommons.unl.edu/physicsrudd

Part of the Physics Commons

Crooks, G. B. and Rudd, M. Eugene, "Experimental Evidence for the Mechanism of Charge Transfer into Continuum States" (1970). M. Eugene Rudd Publications. 63.

https://digitalcommons.unl.edu/physicsrudd/63

This Article is brought to you for free and open access by the Research Papers in Physics and Astronomy at DigitalCommons@University of Nebraska - Lincoln. It has been accepted for inclusion in M. Eugene Rudd Publications by an authorized administrator of DigitalCommons@University of Nebraska - Lincoln. 


\title{
Experimental Evidence for the Mechanism of Charge Transfer into Continuum States
}

\author{
G. B. Crooks and M. E. Rudd \\ Behlen Laboratory of Physics, University of Nebraska, Lincoln, Nebraska 68508
}

Received 2 November 1970

We have observed a prominent peak in the energy spectrum of electrons ejected in the forward direction from helium bombarded by protons ranging in energy from 100 to $300 \mathrm{keV}$. The peak occurs at an ejected-electron velocity equal to the velocity of the incident proton. The experimental results verify the existence of the mechanism of charge transfer into continuum states of the incident ion.

Physical Review Letters 25, 1599 - 1601 (1970)

(C)1970 The American Physical Society

URL: http://link.aps.org/doi/10.1103/PhysRevLett.25.1599

DOI: 10.1103/PhysRevLett.25.1599 


\title{
PHYSICAL REVIEW LETTERS
}

\begin{tabular}{llc}
\hline \hline Volume 25 & 7 DECEMBER 1970 & Number 23 \\
\hline \hline
\end{tabular}

\section{Experimental Evidence for the Mechanism of Charge Transfer into Continuum States*}

\author{
G. B. Crooks and M. E. Rudd \\ Behlen Laboratory of Physics, University of Nebraska, Lincoln, Nebraska 68508 \\ (Received 2 November 1970)
}

\begin{abstract}
We have observed a prominent peak in the energy spectrum of electrons ejected in the forward direction from helium bombarded by protons ranging in energy from 100 to 300 $\mathrm{keV}$. The peak occurs at an ejected-electron velocity equal to the velocity of the incident proton. The experimental results verify the existence of the mechanism of charge transfer into continuum states of the incident ion.
\end{abstract}

Experimental data of Rudd and Jorgensen ${ }^{1}$ and of Rudd, Sautter, and Bailey ${ }^{2}$ show definite humps in energy spectra of electrons ejected at $10^{\circ}$ in collisions of protons on gases. These occur approximately where the ejected-electron velocity is equal to the velocity of the incident ion. This led to the development of theoretical treatments ${ }^{3,4}$ which predict a very strong peak in the energy spectrum of electrons ejected in the forward $\left(0^{\circ}\right)$ direction. We have now recorded such peaks in spectra from 100 - to $300-\mathrm{keV}$ proton impacts on helium gas. The measured cross sections are in agreement with the theory of Macek. ${ }^{3}$

The Born approximation fails to predict this peak and also does not reproduce the cross section even approximately over a large energy region on either side of the peak. This is a case in which the Born theory predicts fewer electrons than observed for ionization by positive particles, but, conversely, it can be expected to predict too many electrons for ionization by negative particles. ${ }^{5}$ This effect has been observed by Heckman and Lindstrom ${ }^{6}$ who find that positive pions lose energy in emulsions faster than negative pions. The difference has been interpreted in terms of the second-order Born approximation. However, the second term of the Born expansion contains no mechanism by which the peak observed in our data can be produced. The Macek theory, which is based on the first term of a Neumann expan- sion of Faddeev's equations, ${ }^{7}$ does contain such a mechanism, namely, charge exchange to a continuum state of the incident proton. This mechanism is described theoretically by selectively summing an infinite number of terms in the Born expansion. ${ }^{7}$ This experiment, which measures electrons ejected at $0^{\circ}$, provides a stringent test of this theory and other theories of ionization.

A simplified schematic of the experimental apparatus is shown in Fig. 1. The proton beam from a Cockcroft-Walton accelerator was collimated by two $0.34-\mathrm{mm}$ apertures spaced $5 \mathrm{~mm}$ apart. A biased aperture at the end of the beam tube prevented secondary electrons generated in the beam tube from reaching the scattering re-

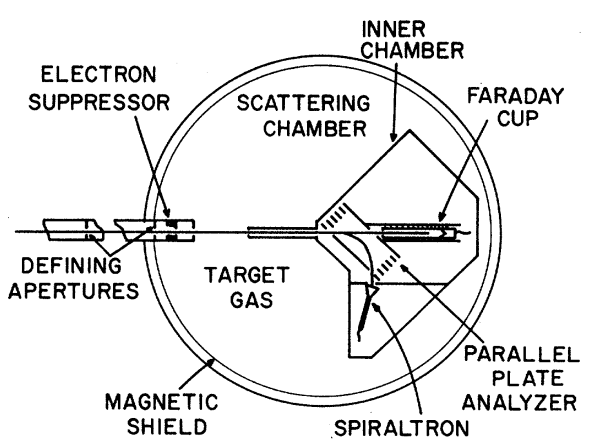

FIG. 1. The experimental arrangement for measuring the ejected-electron spectrum in the forward direction. 
gion. The protons interact with the target gas in the outer chamber and enter the inner chamber with the forward ejected electrons through a 1 $\mathrm{mm}$ slit at the end of an electron pipe. Here both the protons and electrons enter a parallel-plate analyzer having an energy resolution of $1.6 \%$. The proton beam exits through a slit in the back plate of the analyzer and is collected by a Faraday cup, while the electrons are energy analyzed and individually counted by a Bendix Spiraltron electron multiplier.

An energy spectrum of the ejected electrons is obtained by sweeping a multichannel analyzer at $4 \mathrm{msec}$ per channel, producing a ramp voltage proportional to the channel number. This voltage is amplified and used as the analyzing voltage on the back plate of the analyzer while the electron counts are stored in their respective channels.

The target gas pressure was maintained at 1 $\times 10^{-3}$ Torr in the outer chamber, while the pressure in the inner chamber was about $6 \times 10^{-6}$ Torr, yielding a differential pumping ratio of about 165 . The base pressure of the system was $1 \times 10^{-6}$ Torr. The entire chamber was shielded from the Earth's magnetic field by a high-perme-

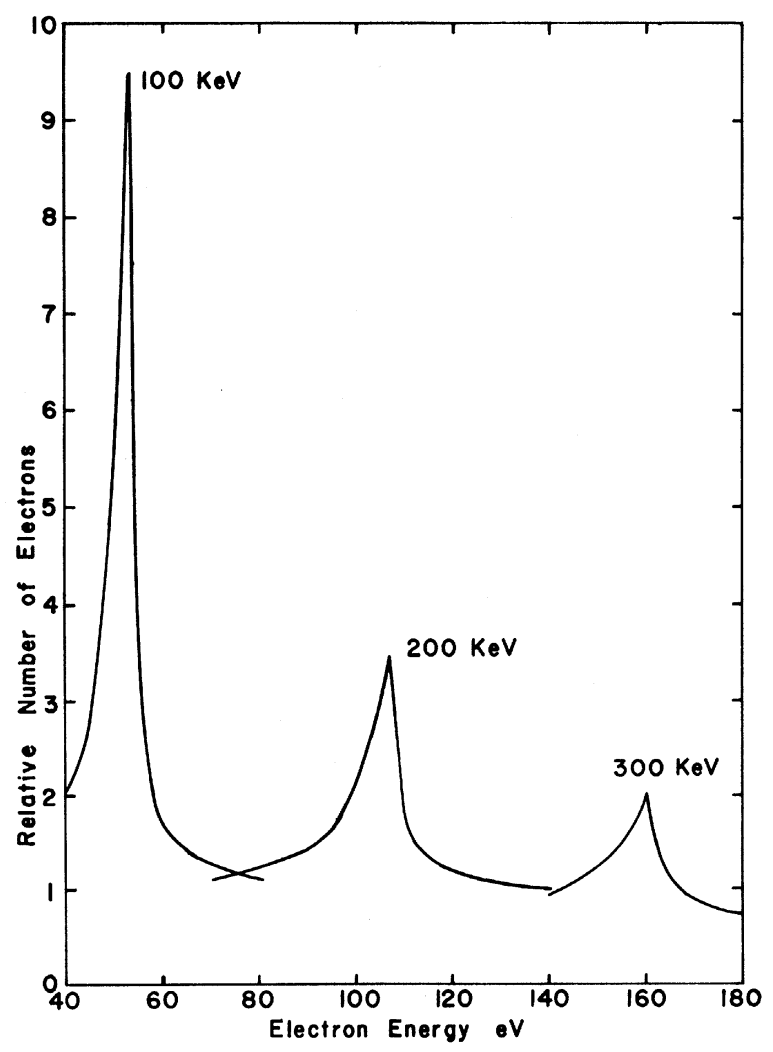

FIG. 2. Electron spectra from three runs at different proton energies. The peaks are at 53, 107, and 160 $\mathrm{eV}$. ability canister.

The shift in energy of the peak with incident proton energy can be seen in Fig. 2. The measured energies at the peaks are within $2 \%$ of that predicted by theory, without being corrected for the effects of contact potentials. The background counts have been subtracted and the three spectra have been normalized to $10.6 \times 10^{-6} \mathrm{C}$ of integrated proton-beam current. The relative heights tend to follow measured charge-exchange cross sections ${ }^{8}$ as one would expect from the mechanism mentioned above. To obtain relative cross sections from these curves, each ordinate must be divided by the electron energy at that point to correct for the variation of analyzer resolution with energy. To put the cross sections on an absolute basis we have normalized at $40 \mathrm{eV}$ to data of Rudd, Sautter, and Bailey ${ }^{2}$ extrapolated to $0^{\circ}$. The results are shown in Fig. 3 along with the Born-approximation predictions for $0^{\circ}$ and the Macek theory for $0^{\circ}$ and $1.4^{\circ}$. The latter angle was chosen because the angular resolution of the apparatus was $\pm 1.4^{\circ}$. The actual results lie be-

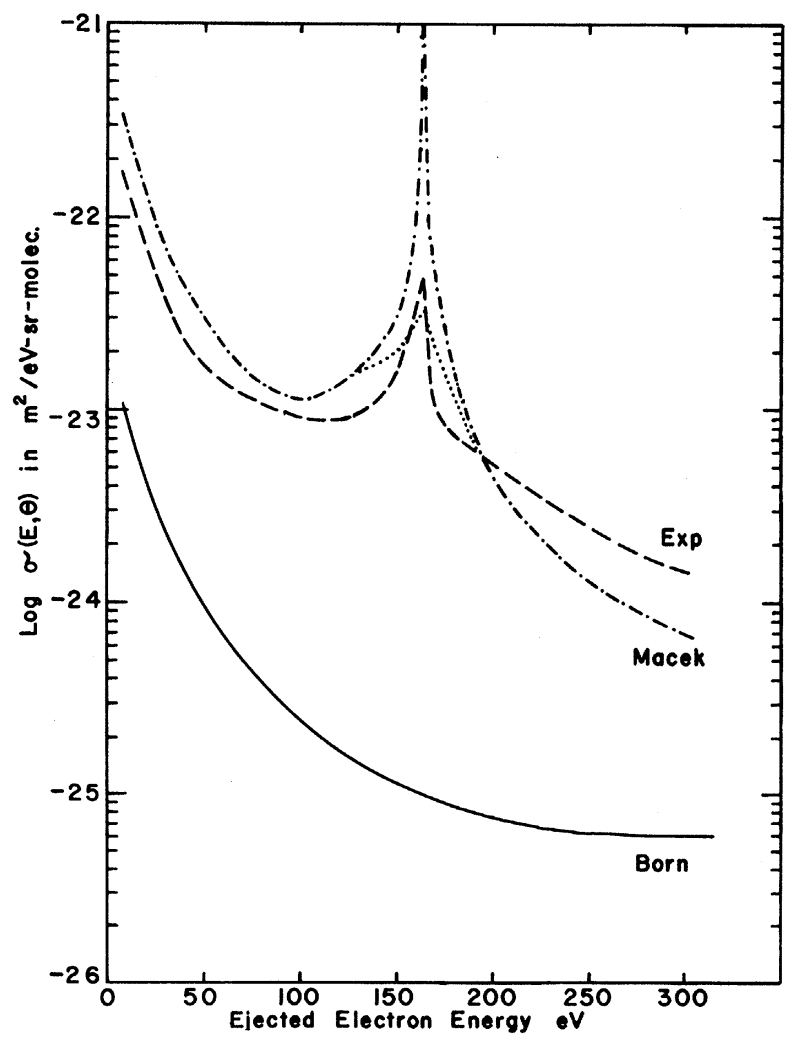

FIG. 3. Cross sections for electrons ejected from He at $0^{\circ}$ by $300-\mathrm{keV} \mathrm{H}^{+}$calculated from experimental data and from two theoretical treatments. Dash-dot curve is Macek theory at $0^{\circ}$; dotted curve at $1.4^{\circ}$. 
tween these two extremes as expected. However, the experimental peak appears to be narrower than expected and also the Macek theory predicts a cross section that is too large for lower energies and too small for the higher energies even when the experimental uncertainty of $30 \%$ is taken into account. The Born results are too low by a factor of 20 .

The peak was also observed using other projectiles and other target gases. It is reasonable to expect that the effect would be appreciable in emulsions and might be useful in explaining the difference in the energy-loss rates of the positive and negative pions. ${ }^{6}$

This experiment provides the first conclusive evidence that charge exchange into continuum states is an important mechanism of ionization by ion impact. Furthermore, in contrast to earlier speculations, ${ }^{9}$ these results show that the Faddeev equations, which provide a natural means of formulating this mechanism in a theory, may apply to the electrostatic interaction.
The authors wish to express their appreciation to Professor J. Macek for the use of his computer programs and for his informative discussions, and to J. Crooks who assisted in taking much of the data.

*Work supported by the National Science Foundation.

${ }^{1}$ M. E. Rudd and T. Jorgensen, Jr., Phys. Rev. 131, 666 (1963).

${ }^{2}$ M. E. Rudd, C. A. Sautter, and C. L. Bailey, Phys. Rev. 151, 20 (1966).

${ }^{3}$ J. Macek, Phys. Rev. A 1, 235 (1970).

${ }^{4}$ A. Salin, J. Phys. B: Proc. Phys. Soc., London $\underline{2}$, 631 (1969).

${ }^{5}$ I. H. Sloan, Proc. Phys. Soc., London, Sect. A $\underline{85}$, 435 (1965).

${ }^{6}$ H. H. Heckman and P. J. Lindstrom, Phys. Rev. Lett. 22, 871 (1969).

${ }^{7}$ L. D. Faddeev, Zh. Eksp. Teor. Fiz. 39, 1459 (1960) [Sov. Phys. JETP 12, 1014 (1961)].

${ }^{8}$ C. F. Barnett and H. K. Reynolds, Phys. Rev. $\underline{109}$, 355 (1958).

${ }^{9}$ D. G. Truhlar, D. C. Cartwright, and A. Kupperman, Phys. Rev. 175, 113 (1968), footnote 99. 